Atsushi Ogawa $\cdot$ Shigenori Yamamoto $\cdot$ Masaki Kanazawa

Masaki Takayanagi $\cdot$ Shuji Hasegawa $\cdot$ Yoichi Kohno

\title{
Identification of two novel mutations of the carnitine/acylcarnitine translocase (CACT) gene in a patient with CACT deficiency
}

\begin{abstract}
Carnitine/acylcarnitine translocase (CACT) transports acylcarnitines into mitochondria in exchange for free carnitine, and is therefore an essential component within the fatty acid beta-oxidation pathway. CACT deficiency is an autosomal recessive disease caused by a mutation of the CACT gene. We have identified two novel mutations of the CACT gene in a patient with CACT deficiency. The first, a deletion mutation (146 del T), leads to premature termination and results in a very immature CACT protein. The second, a splicing mutation (261$10 \mathrm{~T}>\mathrm{G}$ ), results in either skipping of exons 3 and 4 , or of exon 3 alone, and leads to truncation of the protein. Each of these mutations is hypothesized to destroy the function of the CACT protein. We propose that each of these mutations of the $C A C T$ gene play a causative role in the disease.
\end{abstract}

Key words Carnitine/acylcarnitine translocase $\cdot C A C T$ gene $\cdot$ Lariat $\cdot$ Branchpoint $\cdot$ Deletion $\cdot$ Exon skipping

\section{Introduction}

Carnitine/acylcarnitine translocase (CACT), also known as the carnitine/acylcarnitine carrier, is a nuclear-encoded protein located in the inner mitochondrial membrane. It catalyzes the transport of acylcarnitine esters from the cytosol to the mitochondrial matrix in exchange for free carnitine (Indiveri et al. 1991). Carnitine/acylcarnitine transport is an essential step in the process of long-chain fatty acid oxidation.

A. Ogawa $(\varangle) \cdot$ S. Yamamoto $\cdot$ M. Kanazawa $\cdot$ Y. Kohno

Department of Pediatrics, Chiba University School of Medicine, 1-8-

1 Inohana, Chuou-ku, Chiba, Chiba 260-8670, Japan.

Tel. +81-43-226-2144; Fax +81-43-226-2145

e-mail: aogawa@pediat3.m.chiba-u.ac.jp

M. Takayanagi

Division of Metabolism, Chiba Children's Hospital, Chiba, Japan

S. Hasegawa

Chiba City Institute of Health and Environment, Chiba, Japan
There is substantial clinical literature concerning CACT deficiency (Brivet et al. 1994, 1996; Huizing et al. 1997; Huizing et al. 1998; Morris et al. 1998; Niezen-Koning et al. 1995, Olpin et al. 1997, Pande et al. 1993, Stanley et al. 1992). The defining features of severely affected patients with a clinical onset during the neonatal period are hypoketotic hypoglycemia, mild hyperammonaemia, variable dicarboxylic aciduria, hepatomegaly with abnormal liver functions, various cardiac symptoms and skeletal muscle weakness.

Recently, $C A C T$ has been cloned in the rat (Indiveri et al. 1997) and in human (Huizing et al. 1997). The gene has been mapped to chromosome 3p21.31 (Viggiano et al. 1997). Characterization of the exon-intron structure reveals that the $C A C T$ gene is organized into nine exons spanning a genomic region of 16.5-kb (Iacobazzi et al. 1998). Mutations in $C A C T$ cDNA have been reported, including single base insertion and small deletions (Huizing et al. 1997; Huizing et al. 1998). It should be noted, however, that there has been only one report concerning mutations in the $C A C T$ gene at the genomic level (Costa et al. 1999). The purpose of the present study was to investigate mutations in the $C A C T$ gene at the genomic level in a patient with CACT deficiency.

\section{Materials and methods}

\section{Materials}

Fibroblasts obtained from a patient with CACT deficiency were purchased from the National Institute of General Medical Sciences (NIGMUS). A detailed clinical profile of the patient has been published elsewhere (Stanley et al. 1992).

DNA sequencing

Cultured skin fibroblasts were used for polyA+RNA isolation. Oligonucleotides were designed based on the cDNA 
sequence (Huizing et al. 1997) at the following nucleotide positions: 42-65 (forward primer, 5'-GGAGTGACAGACGGACTGACCATG-3'); 978-1003 (reverse primer, 5'CAGCATCCAGAAGTGAACTTGAGCAG-3'). These primers were used to amplify the entire coding sequence by reverse transcriptase polymerase chain reaction (RT-PCR). The cycling conditions were as follows: $95^{\circ} \mathrm{C}$ for $3 \mathrm{~min}$, followed by 30 cycles at $95^{\circ} \mathrm{C}$ for $30 \mathrm{~s}, 60^{\circ} \mathrm{C}$ for $30 \mathrm{~s}$, and $72^{\circ} \mathrm{C}$ for $1 \mathrm{~min}$, with a final extension step of $5 \mathrm{~min}$. The amplified segment was cloned into a pGEM-T easy vector (Promega, Madison, WI, USA) and sequenced using a DNA sequencer (DSQ 2000L; Shimadzu, Kyoto, Japan).

\section{Results}

Amplification of the entire coding sequence of the sample $C A C T$ cDNA using RT-PCR yielded four products. Three were smaller than would be predicted from a sequence of comparable length, while the fourth product was of the expected size (Fig. 1). Each fragment was cloned and sequenced.

A single base deletion, 146 del T, was found in the fragment of the expected size (962-bp). We could not identify a normal sequence clone from this fragment. The $146 \mathrm{del} \mathrm{T}$ mutation was additionally confirmed from the results of a sequencing analysis of the genomic DNA. Exon 1 of the normal $C A C T$ gene, which contains the nucleotide at position 146, was amplified using a primer pair described in Iacobazzi et al. 1998, and sequenced. The deletion mutation, $146 \mathrm{del} \mathrm{T}$, was found in four out of eight of the clones (Fig. 2a).

The results of the analysis of the transcripts derived from the three smaller fragments were as follows: exon 3 was skipped in the transcript in the first of the fragments

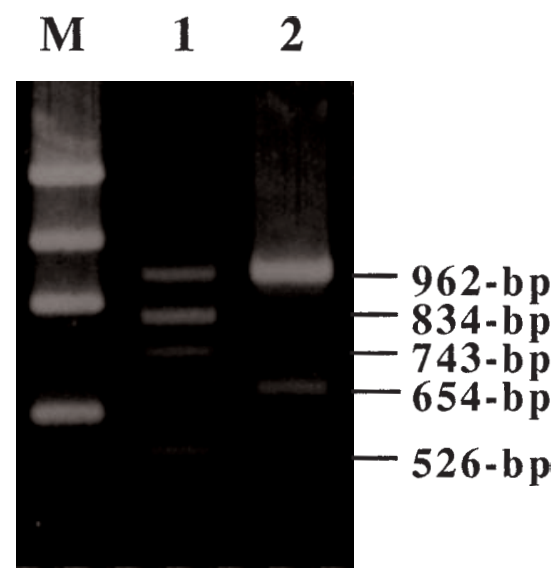

Fig. 1. Analysis of the reverse transcriptase polymerase chain reaction (RT-PCR) products derived from $C A C T$ cDNA from a CACT-deficient patient (1) and from control samples (2). Three smaller fragments are observed, in addition to the fragment of the expected length in the RTPCR products derived from the CACT-deficient patient cDNA. In addition, a smaller fragment (654-bp) is observed in the control data, reflecting a deletion of exons 6,7 and 8 in the $C A C T$ gene. $M, \phi \mathrm{X} 174 /$ HaeIII marker (834-bp). Both exons 3 and 4 were skipped in the second fragment (743-bp), while exons $3,6,7$ and 8 were skipped in the third fragment (526-bp). We could not find any evidence of the aforementioned 146 del T mutation in any of the smaller fragments. In order to identify the mutation causing the skipping of either exon 3 , or both exons 3 and 4 , we amplified and sequenced exons 2,3 and 4 of the $C A C T$ gene from the patient. A single nucleotide change, 261$10 \mathrm{~T}>\mathrm{G}$, was found upstream from a splice acceptor site adjacent to exon 3 in two out of eight of the clones (Fig. 2b). Because the 261-10T $>$ G mutation was found in only two out of eight clones, further confirmation of this mutation was achieved on the basis of restriction enzyme digestion analysis (Fig. 3).

There were no nucleotide changes in either the 5 '-end or the 3 '-end of the exon-intron junction of exons 2 and 4 . A

\section{$\mathbf{a}$}

TCGTCGGTCACCCTCT

control

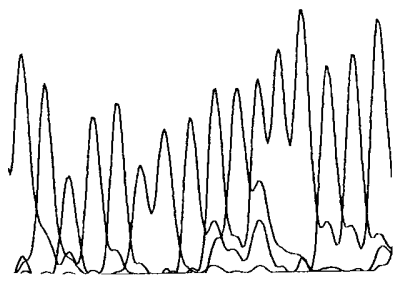

TCGTCGGCACCCTCTG

patient

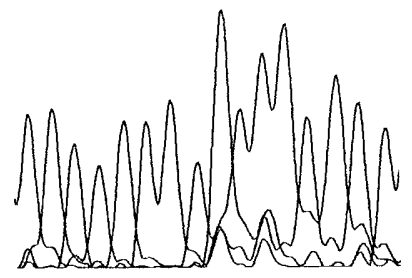

b

\section{TCTGTGATTCCTTGCAG}

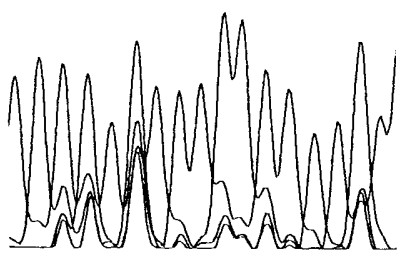

TCTGTGAGTCCTTGCAG

\section{patient}

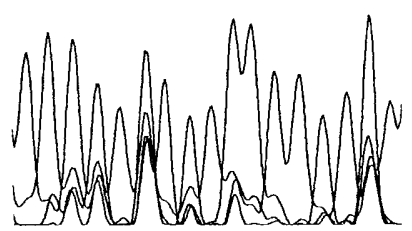

Fig. 2. a, b. Sequencing results of the genomic DNA from the CACTdeficient patient and the control samples. a Underlined letter indicates $\mathrm{T}$ 146, which was deleted in the patient sequence. b Underlined letter indicates the identified nucleotide substitution, 261-10T $>\mathrm{G}$ 


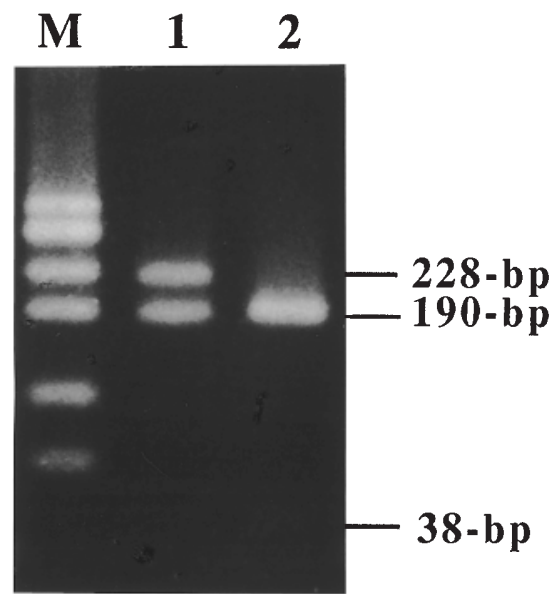

Fig. 3. Exon 3, including the $5^{\prime}$-end of exon-intron junction, which contains the nucleotide position 261-10, was amplified using a primer pair described in Iacobazzi et al.1998. The PCR products were digested with $T f i$ I and were then subjected to electrophoresis on 3\% NuSieve 3:1 agarose gels (FMC BioProducts, Rockland, ME, USA). The Tfi I digestion of the PCR product from the genomic DNA of a normal control generated two DNA fragments of 190 and 38-bp (lane 2). The mutation, 261-10T $>\mathrm{G}$, is known to remove this $T f i$ I site. The Tfi I digestion of the PCR product from the patient generated a DNA fragment of 228-bp derived from a mutated allele that lacks the $T f i$ I site, in addition to 190- and 38-bp (lane 1). This result indicates that this patient is heterozygous for the 261-10T $>\mathrm{G}$ mutation. The 38-bp fragment is barely visible under these conditions. $M, \phi \mathrm{X} 174 /$ Hae III marker

base substitution within this region is known to destroy a $T f i$ I site. We therefore searched for a single nucleotide change among the normal Japanese population, using restriction enzyme digestion. We could not identify a nucleotide change of this kind in any of the 94 alleles of chromosome 3 obtained from normal Japanese control subjects (data not shown). This suggests that this nucleotide change does not represent a polymorphism in the gene. Finally, transcriptional skipping of exons 6,7 and 8 was observed in the control samples (654-bp). Therefore, we chose not to survey the genomic DNA sequence within this area.

\section{Discussion}

We analyzed the $C A C T$ gene in a CACT-deficient patient, and report the existence of two novel mutations. This is the second study to report direct evidence of a molecular defect in CACT genomic DNA. The first of the two mutations, 146 del $\mathrm{T}$, led to a shift in the reading frame such that the mutant protein would have a completely different amino acid sequence from codon 28 to the premature stop codon at position 128. The gene product arising from such a mutation would be unlikely to function as a carrier protein. We propose, therefore, that this deletion mutation in the $C A C T$ gene could confer the disease state observed in the patient.

The second mutation we identified in the CACT gene involved a single nucleotide change, 261-10T $>$ G, upstream from a splice acceptor site adjacent to exon 3 . The mutation was found in two out of eight of the clones. The sequence surrounding the thymidine -10 residue upstream of the normal acceptor splice site, CTGTGAT (-16 to -10$)$, almost matched that of the consensus branchpoint sequence YNYYRAY (Y, pyrimidine; R, purine; $\mathrm{N}$, any base) reported by other investigators (Zeitlin and Efstrastiadis 1984, Ruskin et al. 1984). In addition the match with the aforementioned consensus branchpoint sequence, the following criteria have been established for identifying putative branchpoint sequences (Ruskin et al. 1984). First of all, the branched nucleotide must be adenine. Secondly, the adenine must be followed by a pyrimidine. The second criterion is based on the fact that a pyrimidine-rich region is almost always found following adenine.

Although the function of pyrimidine in the $3^{\prime}$-end of the consensus branchpoint sequence has not been clearly defined, pyrimidine appears to have an important role in lariat formation during pre-mRNA splicing. In the present patient, the pyrimidine (T) in the $3^{\prime}$-end of the consensus sequence changed to purine $(\mathrm{G})$. Under these conditions, it is likely that lariat formation would disappear. We propose, therefore, that the nucleotide change $261-10 \mathrm{~T}>\mathrm{G}$ is the mutation responsible for the exon skipping observed in the present patient.

We observed this characteristic exon skipping in the transcripts derived from the smaller fragments amplified by RT-PCR. Our analysis indicates that the mutation leading to the skipping of exon 3 would lead to a shift in the reading frame, resulting in the production of an immature protein. It should be noted that the mutation in which both exons 3 and 4 are skipped would not lead to a change in the reading frame. However, we predict that such a mutation could still affect the capacity of the mutant gene product to function as a carrier protein. Exons 3 and 4 encode the second and third transmembrane domains, respectively. As described above, the splicing mutation, 261-10T $>\mathrm{G}$, would lead to a truncated gene product. We propose, therefore, that the base substitution $261-10 \mathrm{~T}>\mathrm{G}$ is the disease-causing mutation conferring CACT deficiency.

Analysis of the transcript derived from the fragments amplified by RT-PCR revealed that the transcript carrying the 146 del T mutation did not display exon skipping. Conversely, the transcript that did display exon skipping did not carry the 146 del T mutation. In the light of these findings, taken together, we propose that the patient whose genomic DNA we have studied may be a compound heterozygote for the $146 \mathrm{del} \mathrm{T}$ and $261-10 \mathrm{~T}>\mathrm{G}$ mutations.

In normal controls, both of the 834-bp and 743-bp fragments were also be slightly seen in Fig. 1. Each fragment was cloned and sequenced and was found to skip exon 3 , or both exons 3 and 4, as observed in the patient. In this analysis of the patient, we were unable to find 834-bp or 743-bp fragments carrying the 146 del T mutation, but we would expect to find a clone, that is both exon skipping and carrying the 146 del T mutation, if we analyzed more of the clones.

In summary, we have identified two novel mutations of the $C A C T$ gene in a patient with CACT deficiency. Each of the mutations is hypothesized to destroy the capacity of the 
resulting gene product to function as a carnitine carrier. We propose, therefore, that each of these mutations of the $C A C T$ gene plays a direct role in the manifestation of the disease.

Acknowledgments This work was supported in part by a grant-in-aid (11670739 to Shigenori Yamamoto) for Scientific Research from the Ministry of Education, Science and Culture of Japan.

\section{References}

Brivet M, Slama A, Ogier H, Boutron A, Demaugre F, Saudubray JM Lemonnier A (1994) Diagnosis of carnitine acylcarnitine translocase deficiency by complementation analysis. J Inherit Metab Dis17:271274

Brivet M, Slama A, Millington DS, Roe CR, Demaugre F, Legrand A, Boutron A, Poggi F, Saudubray JM (1996) Retrospective diagnosis of carnitine-acylcarnitine translocase deficiency by acylcarnitine analysis in the proband Guthrie card and enzymatic studies in the parents. J Inherit Metab Dis 19:181-184

Costa C, Costa JM, Nuoffer JM, Slama A, Boutron A, Saudubray JM, Legrand A, Brivet M (1999) Identification of the molecular defect in a severe case of carnitine-acylcarnitine carrier deficiency. J Inherit Metab Dis 22:267-270

Huizing M, Iacobazzi V, Ijlst L, Savelkoul P, Ruitenbeek W, van den Heuvel L, Indiveri C, Smeitink J, Trijbels F, Wanders R, Palmieri F (1997) Cloning of the human carnitine-acylcarnitine carrier cDNA and identification of the molecular defect in a patient. Am J Hum Genet 61:1239-1245

Huizing $M$, Wendel $U$, Ruitenbeek W, Iacobazzi V, IJlst L, Veenhuizen P, Savelkoul P, van den Heuvel LP, Smeitink JA, Wanders RJ, Trijbels JM, Palmieri F (1998) Carnitine-acylcarnitine carrier deficiency: identification of the molecular defect in a patient. J Inherit Metab Dis 21:262-267
Iacobazzi V, Naglieri MA, Stanley CA, Wanders RJ, Palmieri F (1998) The structure and organization of the human carnitine/acylcarnitine translocase (CACT1) gene2. Biochem Biophys Res Commun 252:770-774

Indiveri C, Tonazzi A, Prezioso G, Palmieri F (1991) Kinetic characterization of the reconstituted carnitine carrier from rat liver mitochondria. Biochim Biophys Acta 1065:231-238

Indiveri C, Iacobazzi V, Giangregorio N, Palmieri F (1997) The mitochondrial carnitine carrier protein: cDNA cloning, primary structure and comparison with other mitochondrial transport proteins. Biochem J 321: 713-719

Morris AA, Olpin SE, Brivet M, Turnbull DM, Jones RA, Leonard JV (1998) A patient with carnitine-acylcarnitine translocase deficiency with a mild phenotype. J Pediatr 132:514-516

Niezen-Koning KE, van Spronsen FJ, Ijlst L, Wanders RJ, Brivet M, Duran M, Reijngoud DJ, Heymans HS, Smit GP (1995) A patient with lethal cardiomyopathy and a carnitine-acylcarnitine translocase deficiency. J Inherit Metab Dis 18:230-232

Olpin SE, Bonham JR, Downing M, Manning NJ, Pollitt RJ, Sharrard MJ, Tanner MS (1997) Carnitine-acylcarnitine translocase deficiency - a mild phenotype. J Inherit Metab Dis 20:714-715

Pande SV, Brivet M, Slama A, Demaugre F, Aufrant C, Saudubray JM (1993) Carnitine-acylcarnitine translocase deficiency with severe hypoglycemia and auriculo ventricular block. Translocase assay in permeabilized fibroblasts. J Clin Invest 91:1247-1252

Ruskin B, Krainer AR, Maniatis T, Green MR (1984) Excision of an intact intron as a novel lariat structure during pre-mRNA splicing in vitro. Cell 38:317-331

Stanley CA, Hale DE, Berry GT, Deleeuw S, Boxer J, Bonnefont JP (1992) Brief report: a deficiency of carnitine-acylcarnitine translocase in the inner mitochondrial membrane. N Engl J Med 327:19-23

Viggiano L, Iacobazzi V, Marzella R, Cassano C, Rocchi M, Palmieri F (1997) Assignment of the carnitine/acylcarnitine translocase gene (CACT) to human chromosome band 3p21.31 by in situ hybridization. Cytogenet Cell Genet 79:62-63

Zeitlin S, Efstratiadis A (1984) In vivo splicing products of the rabbit beta-globin pre-mRNA. Cell 39:589-602 